\title{
Relações entre índices preço-lucro e retornos dos títulos públicos
}

(Relationships between price-earnings ratios and returns of treasury bonds)

\author{
Daniel Penido de Lima Amorim ${ }^{\dagger}$ \\ Marcos Antônio de Camargos
}

\begin{abstract}
Resumo Os índices P/E1 e P/E10 ou Índice Preço-lucro Ciclicamente Ajustado foram amplamente disseminados na literatura que tem como contexto o mercado de ações americano. Este artigo traz um método de construção de índices P/E para o mercado de ações brasileiro. Seu objetivo é analisar as relações de longo prazo entre tanto o P/E1 quanto o P/E10 e as taxas de juros correspondentes aos retornos de títulos públicos, de modo a testar a relação referente ao Modelo Fed. No geral, o período considerado foi de dezembro de 2004 a junho de 2018. Foram estimados modelos Autorregressivos de Defasagens Distribuídas, os quais podem ser representados como Modelos de Correção de Erros Condicionais. Os resultados evidenciaram relações de longo prazo significativas entre tanto o P/E1 quanto P/E10 e as referidas taxas de juros, sugerindo que o Modelo Fed está em linha com o comportamento do mercado financeiro brasileiro.
\end{abstract}

Palavras-chave: Finanças comportamentais; Índice preço-lucro; Modelo Fed; Títulos públicos.

Código JEL: G10, G11, 12.

\begin{abstract}
The ratios P/E1 and P/E10 or the cyclically adjusted price-to-earnings ratio are widely disseminated in the literature based on the U.S. stock market. This paper introduces a method to construct $\mathrm{P} / \mathrm{E}$ ratios for the Brazilian stock market. The purpose of this paper is to analyze the long-term relationships between both P/E1 and P/E10 and interest rates corresponding to the returns of treasury bonds, in order to test the Fed Model. In general, the period considered was from December 2004 to June 2018. Autoregressive distributed lags models were estimated, which can be represented as conditional error correction models. Results show significant long-term relationships between both P/E1 and P/E10 and the relevant interest rates, suggesting that the Fed Model is in line with the behavior of the Brazilian financial market.
\end{abstract}

Keywords: Behavioral finance; Price-earnings ratio; Fed Model; Treasury bonds.

JEL Code: G10, G11, 12.

\section{Introdução}

As taxas de juros estão entre os fatores mais associados ao nível de preços no mercado de ações. Shiller (2015) discute uma suposta relação negativa en-

Submitted on June 6, 2020. Revised on August 3rd, 2020. Accepted on August 3rd, 2020. Published online in September 2020. Editor in charge: Marco Lyrio.

$\dagger$ Universidade Federal de Minas Gerais, Brazil: daniel_amorim23@hotmail.com

*Universidade Federal de Minas Gerais e Faculdade IBMEC de Belo Horizonte, Brazil:

marcosaceface.ufmg.br 
tre um índice $\mathrm{P} / \mathrm{E}$ do mercado americano e as taxas de juros. O P/E em questão é conhecido como Índice Preço-lucro Ciclicamente Ajustado ${ }^{1}$ (CAPE), consistindo na razão entre o Índice Standard Poor's (S\&P) 500 e uma média móvel de dez anos do Índice S\&P 500 Earnings - um índice de lucros das empresas cujas ações compõem o referido índice de preços. A relação entre esse P/E e as taxas de juros tem embasamento na ideia de que o investimento no mercado de títulos públicos é uma alternativa àquele no mercado de ações. Nesse sentido, na medida em que o Federal Reserve (Fed) reduzisse as taxas de juros e os Treasury Bonds - títulos do Tesouro dos Estados Unidos cujos retornos são atrelados às taxas de juros pós-fixadas - passassem a oferecer retornos menores, os investidores deixariam de aplicar no mercado de títulos para aplicar no mercado de ações. Logo, um aumento na procura por ações impactaria os preços desses ativos, por conseguinte, a razão P/E. O inverso poderia ser dito quando o Fed aumentasse as taxas de juros. O fluxo de capitais entre o mercado de títulos e o mercado de ações levaria a um equilíbrio de longo prazo entre esses dois mercados.

A ideia de que as taxas de juros explicam o nível dos preços no mercado de ações foi amplamente difundida nos Estados Unidos no final da década de 1990, em virtude de, nessa época, ter ocorrido uma forte valorização dos ativos enquanto as taxas de juros caíam. Nesse contexto, um relatório de política monetária do Fed alegadamente teria comentado a aparente relação negativa entre um índice $\mathrm{P} / \mathrm{E}$ e as taxas de juros, fazendo com que tal relação ficasse conhecida como Modelo Fed. Outrossim, entre meados da década de 1960 e o início da década de 1980, as taxas de juros estavam subindo, enquanto o $\mathrm{P} / \mathrm{E}$ do mercado americano estava caindo, o que fez com que a referida relação parecesse fazer sentido (Weigand e Irons, 2008; Shiller, 2015). A Figura 1 ilustra as séries temporais desses indicadores para os Estados Unidos no período de janeiro de 1881 a junho de 2018.

Todavia, Shiller (2015) alega que a relação entre o P/E e as taxas de juros, a qual corresponde ao Modelo Fed, é fraca. De fato, o coeficiente de correlação de Pearson para todo o período mostrado no Figura 1 é de apenas -0,16. $\mathrm{O}$ autor argumenta que, em outros momentos, a relação não se manifestou, o que pode ser observado nesse gráfico. Ele destaca que, após a eclosão da Crise de 1929, assim como após o estouro da bolha especulativa das empresas ponto-com, em 2001, essa relação praticamente não existia. Nesses momentos, apesar de as taxas de juros terem sido baixas, elas não eram acompanhadas por altos preços das ações. Na perspectiva de Shiller (2015), "embora as taxas de juros devam ter algum efeito sobre o mercado, os preços das ações não mostram nenhuma relação simples ou consistente com as taxas de juros"

${ }^{1}$ Tradução de Cyclically Adjusted Price-to-Earnings (CAPE) Ratio. 


\section{Figura 1 \\ Índice P/E10 e taxa de juros de longo prazo dos treasury bonds de 10 anos nos Estados Unidos}

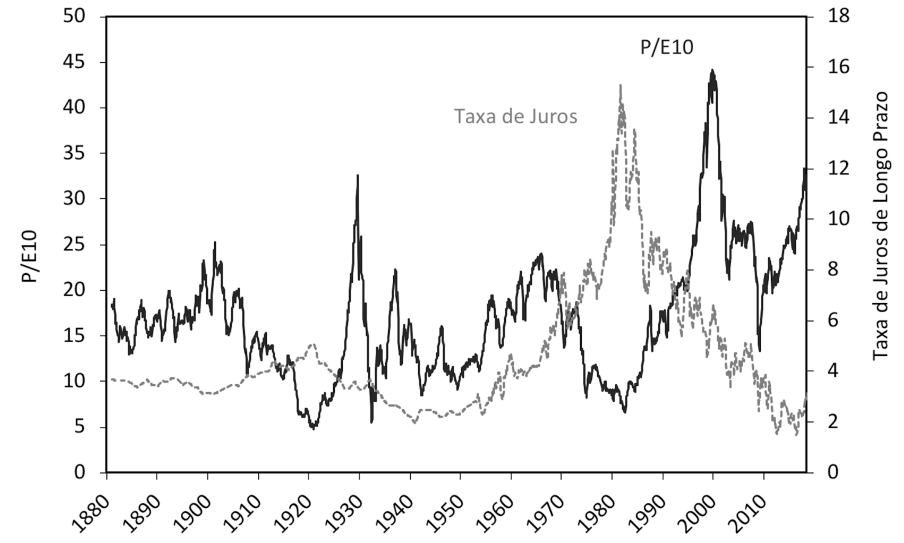

Fonte: http://www.econ.yale.edu/ shiller/data.htm

(Shiller, 2015, p. 12).

Asness (2003) analisou a relação entre o P/E do mercado americano e os retornos dos Treasury Bonds, ainda que se concentrasse mais na relação entre tais retornos e o earnings yield $(\mathrm{E} / \mathrm{P})$ - a razão inversa àquela do $\mathrm{P} / \mathrm{E} .^{2}$ Esse autor discutiu o fato de esse modelo relacionar o $\mathrm{E} / \mathrm{P}$ ou o $\mathrm{P} / \mathrm{E}$, ambos em valores reais, com taxas de juros nominais correspondentes aos retornos dos títulos. Segundo Asness (2003), isso implicaria em um efeito chamado ilusão monetária, o qual consiste no fato de as variações na taxa de inflação não serem levadas em consideração pelos investidores. Porém, Asness (2003) evidencia que, mesmo incorrendo nesse viés em suas análises, os investidores guiaram a trajetória do P/E como uma função da taxa de juros nominal. Quanto à ilusão monetária, Shiller (2015) reitera que, comumente, os indicadores de retornos no mercado de ações são apresentados na mídia em termos de valores nominais, portanto, as pessoas podem naturalmente acreditar que esses retornos nominais devam continuar no futuro.

Segundo Asness (2003), no mercado financeiro americano, entre 1965 e 2001, as taxas de juros foram baixas (altas), enquanto o E/P foi baixo (alto), ou, de forma análoga, enquanto o P/E foi alto (baixo). Ou seja, com base no

\footnotetext{
${ }^{2}$ Asness (2003) também analisou a capacidade de o Modelo Fed prever os retornos futuros das ações, relacionando esses retornos ao E/P e às taxas de juros dos Treasury Bonds em regressões. A evidência empírica provida por esse autor sugeriu que o Modelo Fed falha nesse quesito. Não se objetivou testar a referida capacidade preditiva no presente estudo.
} 
comportamento do mercado financeiro, durante esse período, seria razoável definir o E/P ou o P/E como função das taxas de juros nominais. Contudo, Asness (2003) argumentou que, para a relação ser efetiva durante todo o período de 1926 a 2001, o modelo também teve que considerar o risco percebido - a volatilidade observada - pelos investidores no mercado de ações e no mercado de títulos. ${ }^{3}$ Quanto aos resultados de Asness (2003), estimando modelos de séries temporais por Mínimos Quadrados Ordinários (MQO), esse autor evidenciou que as variáveis do Modelo Fed foram altamente relacionadas entre 1965 e 2001, independentemente de o modelo incorporar variáveis de volatilidade no mercado de ações e no mercado de títulos. Contudo, a referida modificação, que diz respeito à consideração da volatilidade nos distintos mercados, foi fundamental para que a relação fosse efetiva quando considerado todo o período de 1926 a 2001. Asness (2003) concluiu que, apesar de sofrer de ilusão monetária, o investidor do mercado financeiro americano, de fato, seguia o Modelo Fed.

Estrada (2006) avaliou a relação entre o índice P/E e os retornos dos títulos públicos, em termos de taxas de juros, em mercados financeiros de vinte países desenvolvidos. Para isso, esse autor utilizou o teste de cointegração de Engle e Granger (1987), que se baseia em um teste de raiz unitária aplicado sobre o resíduo de uma regressão entre as variáveis de interesse. Os resultados indicaram que havia cointegração entre as variáveis em apenas dois dos países. Todavia, é importante destacar que Estrada (2006) não incluiu as variáveis de volatilidade nas equações do teste, ignorando a sugestão de Asness (2003), o que deve ter influenciado consideravelmente os resultados encontrados. $^{4}$

Cabe destacar que Shiller $(1989,2005)$ proveu tanto um P/E calculado com dados contemporâneos de indicadores de preços de ações e de lucros acumulados anualmente, o P/E1, bem como um P/E que traz como divisor uma média que considera dados dos últimos 10 anos de um indicador de lucros gerados pelas empresas, o P/E10. Em geral, a literatura tem analisado efeitos sobre o P/E10 quando trata do Modelo Fed. Este artigo utiliza-se de uma adaptação do método de Shiller $(1989,2005)$ para a construção de um P/E1 e um P/E10 baseados na carteira teórica do Ibovespa. Essa abordagem supera algumas dificuldades do contexto brasileiro, como os índices de preços de ações da bolsa de valores brasileira — B3 (Brasil, Bolsa, Balcão) -

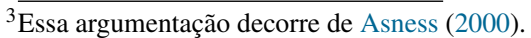

${ }^{4}$ Os vinte países cujos mercados financeiros foram analisados em Estrada (2006) são: Austrália, Áustria, Bélgica, Canadá, Dinamarca, Finlândia, França, Alemanha, Irlanda, Japão, Itália, Holanda, Nova Zelândia, Noruega, Portugal, Espanha, Suécia, Suíça, Reino Unido e Estados Unidos. Os países nos quais encontrou-se cointegração foram Irlanda e Nova Zelândia.
} 
reincorporarem proventos, o que os tornam não adequados para o cálculo dos $\mathrm{P} / \mathrm{Es}$, bem como a ausência de um índice de lucros.

Nesse contexto, o objetivo deste artigo consiste em avaliar as relações entre os índices $\mathrm{P} / \mathrm{E}$ do mercado de ações brasileiro e os retornos dos títulos públicos. A análise dessas relações, que correspondem ao Modelo Fed, é importante para uma melhor compreensão da trajetória dos índices P/E1 e $\mathrm{P} / \mathrm{E} 10$ baseados no Ibovespa, quando o mercado for avaliado com base nesses múltiplos. Não são conhecidos estudos que analisassem os efeitos de longo prazo das taxas de juros sobre os $\mathrm{P} / \mathrm{Es}$, mesmo considerando tanto o contexto do mercado financeiro brasileiro quanto aquele americano.

A abordagem de análise de relações cointegradas de Pesaran et al. (2001) foi empregada para avaliar as relações entres os índices $\mathrm{P} / \mathrm{E}$ e os retornos dos títulos públicos. Essa abordagem parte da estimação de modelos Autorregressivos de Defasagens Distribuídas (ARDL), que podem ser representados como Modelos de Correção de Erros Condicionais (CECM), permitindo, assim, estimar os efeitos de longo prazo da variável de retornos dos títulos públicos sobre os P/Es. Informações a respeito das relações de longo prazo são importantes, especialmente, para investidores que adotam estratégias de investimento de longo prazo, em contraste com aquelas dos efeitos transitórios que pouco devem interessá-los.

Após esta Introdução, a Seção 2 descreve o método de construção dos índices P/E; a Seção 3 descreve os métodos adotados na análise empírica do Modelo Fed; a Seção 4 apresenta os resultados encontrados; e a Seção 5 tece as considerações finais.

\section{Construção dos índices preço-lucro}

\subsection{Dados}

Para a construção das séries históricas dos índices apresentados neste estudo, utilizou-se de dados referentes a todas as ações que participaram da carteira teórica do Ibovespa no período de dezembro de 2004 a junho de 2018, a saber: i) cotações de fechamento do último dia do mês, ajustadas por splits/inplits e proventos; ii) ponderações correspondentes às participações percentuais atribuídas, no referido índice, a cada ação no último dia do mês; iii) lucros por ação trimestrais. Esses dados foram coletados pela internet no sistema de informações financeiras Comdinheiro (https : / / www. comdinheiro.com.br).

No Brasil, a intensificação da publicação dos demonstrativos financeiros trimestrais, que trazem dados de lucro por ação, só veio a acontecer a partir de meados da década de 2000. Em virtude disso, dezembro de 2004 foi a data 
estabelecida como início das séries dos indicadores utilizados neste artigo. No momento da coleta, havia dados disponíveis até junho de 2018. Ao todo, 157 ações participaram da composição do Ibovespa nos 162 meses que vão de dezembro de 2004 a junho de 2018.

\subsection{Construção do índice de preços de ações}

Uma das questões metodológicas em relação aos P/Es adaptados ao mercado brasileiro consiste na escolha de um índice de preços de ações amplo tomado como referência para a construção desses múltiplos. Apesar de o Ibovespa agrupar uma menor diversidade de ações quando comparado ao Índice Brasil 100 (IBrX100) e ao Índice Brasil Amplo (IBrA), o mesmo foi adotado em virtude de ser historicamente a maior referência do comportamento do mercado brasileiro. Esse é o mais antigo índice da bolsa de valores brasileira, implementado em 1968 (Castro et al., 2019).

O Ibovespa apresenta uma metodologia que difere daquela do S\&P 500 utilizado por Shiller $(1989,2005)$ no cálculo de seus P/Es. Ele consiste em um índice de retorno total, isto é, seu cálculo considera retornos decorrentes tanto da variação dos preços das ações quanto da distribuição de proventos dividendos, juros sobre capital próprio, direitos de subscrição, entre outros (BM\&FBovespa, 2014). Por outro lado, o S\&P 500 utilizado pelo referido autor consiste em um índice de retorno de preços, isto é, seu cálculo considera retornos restritos aos ganhos e perdas de capital decorrentes das variações dos preços das ações (S\&P Dow Jones Indices, 2018). Em virtude de incorporar o reinvestimento de todas as mencionadas maneiras de distribuição de caixa aos acionistas, os índices de retorno total, como o Ibovespa, tendem a apresentar retornos positivos maiores, os quais, por sua vez, devem implicar uma trajetória mais ascendente do que aquelas de índices que consideram somente os retornos em termos de preços das ações, como o S\&P 500 de retorno de preços. ${ }^{5}$ No cálculo de P/Es, o índice de preços de ações deve ser, de fato, um indicador de preços, não de todos os retornos para o acionista, tendo em vista que a finalidade de tais índices é comparar os preços de ações com os lucros gerados pelas empresas.

Portanto, um método de cálculo de P/Es com dados do mercado brasileiro deve partir da construção de um índice de preços de ações, o qual deve considerar os retornos apenas em termos de preços dos ativos, ainda que possa considerar as ponderações do Ibovespa. Procedeu-se o cálculo desse índice

\footnotetext{
${ }^{5}$ Existe tanto um Índice S\&P 500 de retorno de preços quanto um S\&P 500 de retorno total. Uma comparação entre os dados desses dois índices permite visualizar a trajetória mais ascendente daquele de retorno total.
} 
de preços de ações, $I_{P}$, conforme a seguinte equação:

$$
I_{P t}=\frac{\sum_{i=1}^{N} P_{i, t} \rho_{i, t}}{\sum_{i=1}^{N} \rho_{i, t}},
$$

na qual $P$ representa o preço de uma ação qualquer $i$ presente na composição do Ibovespa, e $\rho$ representa a ponderação correspondente à participação percentual dessa ação $i$ nesse índice tomado como referência.

Como mencionado, o I $\mathrm{I}_{\mathrm{P}}$ segue as mesmas ponderações da carteira teórica do Ibovespa, as quais variam conforme a capitalização das ações ao longo do tempo, ainda que dentro de certos limites. As ponderações da carteira teórica do Ibovespa variam diariamente, apesar de haver revisão da composição da carteira somente de quatro em quatro meses. O rebalanceamento do IP ocorre em função da variação mensal dessas ponderações. Esse rebalanceamento considera também a inclusão ou exclusão de ações na carteira do Ibovespa. Contudo, diferentemente do Ibovespa, medido em pontos, por tratar-se de uma média ponderada dos preços das ações, o $I_{P}$ é medido em valor monetário.

\subsection{Construção do índice de lucros por ação}

Diferentemente do Índice S\&P 500, que tem os lucros das ações que participam de sua composição refletidos pelo S\&P 500 Earnings, o Ibovespa não dispõe de um índice que sirva como referência em termos de lucros por ação. Sendo assim, para a construção de P/Es com dados do mercado brasileiro, um índice de lucros por ação deve ser construído.

Procedeu-se o cálculo desse índice de lucros por ação, LPA, conforme a seguinte equação:

$$
I_{\mathrm{LPA} t}=\frac{\sum_{i=1}^{N} \mathrm{LPA}_{i, t} \rho_{i, t}}{\sum_{i=1}^{N} \rho_{i, t}},
$$

na qual LPA representa o lucro por ação referente a uma ação qualquer $i$ que faz parte da composição do Ibovespa, e $\rho$ representa a ponderação correspondente à participação percentual dessa ação nesse índice de preços de ações tomado como referência.

Como o I IPA inicialmente é um parâmetro trimestral, em virtude de os dados de lucro por ação serem publicados trimestralmente, seguindo o método de Shiller (2005), utiliza-se de interpolação linear para construir uma série mensal desse indicador. $\mathrm{O}_{\text {LPA }}$ consiste em uma média ponderada dos lucros por ação, sendo mensurado em termos de valor monetário. Seu rebalanceamento é semelhante ao do $\mathrm{I}_{\mathrm{P}}$, considerando as ponderações da carteira teórica do Ibovespa. 


\subsection{Cálculo dos índices preço-lucro}

Como mencionado, Shiller $(1989,2005)$ proveu dois P/Es distintos: o P/E1 e o P/E10 — também conhecido como CAPE. Enquanto Shiller (1989) calcula P/Es de periodicidade anual, Shiller (2005) calcula tais índices de periodicidade mensal. Utilizando dos dados do $\mathrm{I}_{\mathrm{P}}$ e do $\mathrm{I}_{\mathrm{LPA}}$, o cálculo dos $\mathrm{P} /$ Es para o mercado de ações brasileiro é realizado seguindo basicamente o método de Shiller (2005). O P/E1, que considera apenas o índice de lucros no período $t$, é expresso pela equação (3):

$$
P / E 1_{t}=\frac{I_{P t}}{I_{\mathrm{LPA} t}} .
$$

O P/E10, que considera como divisor uma média retroativa do índice de lucros gerados nos dez anos imediatamente anteriores, é expresso pela equação (4):

$$
P / E 10_{t}=\frac{I_{P t}}{I_{\mathrm{LPA}_{t}}+I_{\mathrm{LPA}_{t-1}}+\ldots+I_{\mathrm{LPA}_{t-119}} / 120} .
$$

Em linha com Shiller (1989, 2005), foram adotados os índices de preços e de lucros em valores reais, sendo eles corrigidos pela inflação com base em junho de 2018. O Índice de Preços ao Consumidor Amplo (IPCA) foi utilizado na correção monetária devido ao fato de o mesmo ser o índice de inflação oficial no Brasil. Os P/Es são calculados mensalmente, seguindo o método de Shiller (2005).

A média retroativa do índice de lucros no P/E10 suaviza as variações desse indicador. Quando decidiu por adotar essa média, Shiller (2005) considerou a afirmação de Graham e Dodd (1934) sobre os lucros das empresas comportarem-se de forma volátil demais para serem analisados anualmente. Essa média torna o P/E10 menos sensível aos descasamentos de curto prazo entre os preços e os lucros, em comparação com o P/E1.

\section{Métodos da análise empírica}

Asness (2003) avaliou se o Modelo Fed era capaz de explicar o comportamento do mercado financeiro americano por meio da estimação de regressões de séries temporais por MQO. Esse autor regrediu a série do $\mathrm{E} / \mathrm{P}$ e a série do $\mathrm{P} / \mathrm{E}$ contra aquela de taxas de juros nominais $(\mathrm{Y})$ - correspondentes aos retornos dos Treasury Bonds de vencimento em 10 anos — em modelos estáticos, isto é, em modelos que não incluíam variáveis defasadas. É importante salientar que a consideração de taxas de juros nominais tem fundamento na ilusão monetária da qual os investidores supostamente sofrem. Asness (2003) 
e Shiller (2015) acreditam que os investidores incorram nesse viés em suas análises.

No contexto do mercado financeiro brasileiro, o Índice de Mercado ANBIMA (IMA-S), referente aos títulos públicos federais cujos retornos são atrelados à taxa SELIC - Tesouro SELIC —, consiste em um indicador adequado para representar o desempenho desses referidos títulos públicos. ${ }^{6}$ Os dados diários e mensais do IMA-S foram coletados no Sistema Gerenciador de Séries Temporais (SGS) do Banco Central do Brasil (https: //www3 . bcb.gov.br/sgspub). Por meio dos dados mensais, foram calculados os retornos no mercado de títulos e, por meio daqueles diários, foi calculada uma medida de volatilidade. Foram utilizados dados diários de fechamento ajustado do Ibovespa para calcular uma medida de volatilidade no mercado de ações. Esses dados foram coletados no sistema de informações financeiras Comdinheiro (http: / / comdinheiro.com.br).

As estimações por MQO adotadas por Asness (2003) implicaram problemas de autocorrelação quando utilizadas para modelar as relações entre os $\mathrm{P} /$ Es e as variáveis relacionadas aos retornos dos títulos públicos no contexto do mercado financeiro brasileiro. Regredindo as séries temporais por MQO, sem incluir variáveis defasadas, defrontou-se com estatísticas Durbin-Watson muito baixas. Granger e Newbold (1974) alertaram para o risco de regressões espúrias quando as estatísticas Durbin-Watson são muito baixas. Os modelos estimados por MQO, com suas estatísticas Durbin-Watson e testes de autocorrelação Breusch-Godfrey, são apresentados no Apêndice. As especificações desses modelos são explicadas a seguir.

\subsection{Modelos autorregressivos de defasagens distribuídas}

Buscando superar o referido problema de autocorrelação, as estimações correspondentes ao Modelo Fed foram realizadas por meio de modelos Autorregressivos de Defasagens Distribuídas (ARDL). Essa classe de modelos dinâmicos tem incluídas na equação a variável dependente defasada e, possivelmente, as variáveis independentes também defasadas. Apesar de modelos de defasagens distribuídas não serem novos na literatura econômica (Almon, 1965; Schmidt, 1974), os modelos ARDL ganharam maior popularidade a partir dos trabalhos de Pesaran e Shin (1998) e Pesaran et al. (2001). Esses autores empregaram modelos ARDL em uma abordagem para testar a existência de relações entre variáveis em nível, o que foi associado à cointegração.

O modelo ARDL correspondente ao Modelo Fed Simples, que não considera as variáveis de volatilidade no mercado de ações e no mercado de títulos,

6 A metodologia dos Índices de Mercado ANBIMA está disponível em: https : / / www . anbi ma.com.br/pt_br/informar/precos-e-indices/indices/ima.htm. 
é representado pela equação (5):

$$
P / E_{t}=\alpha_{0}+\sum_{i=1}^{p} \beta_{1, i} P / E_{t-i}+\sum_{i=0}^{q_{1}} \beta_{2, i} \mathrm{IMAS}_{t-i}+\varepsilon_{t},
$$

na qual $\alpha_{0}$ consiste em uma constante; $P / E$ consiste em um índice preçolucro baseado no Ibovespa, podendo ser o P/E1 ou o P/E10; IMAS consiste nos retornos do índice de títulos Tesouro SELIC; e $\varepsilon_{t}$ consiste no termo de erro da regressão. Além disso, $p$ e $q$ denotam a estrutura do modelo no que concerne às defasagens.

Segundo Asness (2000, 2003), é importante que a especificação correspondente ao Modelo Fed também incorpore variáveis referentes ao risco volatilidade - no mercado de títulos e no mercado de ações. Com isso, o Modelo Fed Simples foi aumentado com essas variáveis. Como proxy da volatilidade no mercado de ações, utilizou-se do desvio padrão mensal do Ibovespa, e, como proxy da volatilidade no mercado de títulos, utilizou-se do desvio padrão mensal do IMA-S. ${ }^{7}$ O Modelo Fed Aumentado é especificado na forma de um modelo ARDL conforme pela equação (6):

$$
\begin{aligned}
P / E_{t}=\alpha_{0} & +\sum_{i=1}^{p} \beta_{1, i} P / E_{t-i}+\sum_{i=0}^{q_{1}} \beta_{2, i} \mathrm{IMAS}_{t-i} \\
& +\sum_{i=0}^{q_{2}} \beta_{3, i} \sigma_{\text {ações }_{t-i}}+\sum_{i=0}^{q_{3}} \beta_{4, i} \sigma_{\text {títulos }_{t-i}}+\varepsilon_{t},
\end{aligned}
$$

em que $\sigma_{\text {ações }}$ consiste na volatilidade no mercado de ações, e $\sigma_{\text {títulos }}$ consiste na volatilidade no mercado de títulos.

Diferentemente de Asness (2003), que utilizou volatilidades do mercado de títulos e do mercado de ações calculadas com base nos dados dos 20 anos passados, neste estudo utilizou-se das volatilidades mensais desses mercados, calculadas sobre os dados diários dos índices subjacentes. Não seria possível considerar períodos tão longos para calcular o desvio padrão dos índices no contexto do mercado financeiro brasileiro, visto que as séries temporais utilizadas neste estudo não são tão longas como aquelas utilizadas em Asness

\footnotetext{
${ }^{7}$ Quando há cointegração, os efeitos de longo prazo são estimados por meio de Modelos de Correção de Erros Condicionais (CECM). Nesses modelos, as variáveis são especificadas em primeira diferença. Com isso, caso a volatilidade fosse calculada como o desvio padrão dos retornos dos índices, ao invés de o desvio padrão dos índices em nível, quando estivessem em primeira diferença nos modelos CECM, elas seriam um mero ruído, que não teria capacidade de explicar os índices P/E. Tentou-se estimar os modelos deste estudo com as medidas de volatilidade calculadas como desvio padrão do índice e como desvio padrão dos retornos, antes de se chegar a essa conclusão.
} 
(2003). Por outro lado, parece mais realístico considerar que o investidor observe a volatilidade do último mês que se passou, ao invés de considerar a volatilidade dos 20 anos passados.

\subsection{Modelos de correção de erros condicionais}

Uma vantagem dos Modelos de Correção de Erros Condicionais (CECM) em relação aos modelos ARDL é que eles dissociam efeitos de curto prazo e efeitos de longo prazo. Os efeitos de longo prazo decorrem dos desvios do equilíbrio de longo prazo entre as variáveis. Logo, havendo cointegração entre as variáveis de interesse, seguindo a abordagem de Pesaran et al. (2001), os modelos ARDL denotados pelas equações (5) e (6) podem ser representados como CECM, sendo eles especificados, respectivamente, como

$$
\begin{gathered}
\Delta P / E_{t}=\alpha_{0}+\sum_{i=1}^{p-1} \beta_{1, i} \Delta P / E_{t-i}+\sum_{i=0}^{q_{1}-1} \beta_{2, i} \Delta \mathrm{IMAS}_{t-i} \\
+\lambda_{1} P / E_{t-1}+\lambda_{2} \mathrm{IMAS}_{t-1}+\varepsilon_{t} \\
\Delta P / E_{t}=\alpha_{0}+\sum_{i=1}^{p-1} \beta_{1, i} \Delta P / E_{t-i}+\sum_{i=0}^{q_{1}-1} \beta_{2, i} \Delta \mathrm{IMAS}_{t-i} \\
+\sum_{i=0}^{q_{2}-1} \beta_{3, i} \Delta \sigma_{\text {ações }_{t-i}}+\sum_{i=0}^{q_{3}-1} \beta_{4, i} \Delta \sigma_{\text {títulos }_{t-i}} \\
+\lambda_{1} P / E_{t-1}+\lambda_{2} \mathrm{IMAS}_{t-1}+\lambda_{3} \sigma_{\text {ações }_{t-1}} \\
+\lambda_{4} \sigma_{\text {títulos }_{t-1}}+\varepsilon_{t}
\end{gathered}
$$

nos quais os coeficientes das variáveis independentes em primeira diferença $(\Delta)$ correspondem aos efeitos de curto prazo sobre um P/E em questão, e os coeficientes $\lambda$ das variáveis independentes correspondem aos efeitos de longo prazo. Os conjuntos de $\lambda$ nas equações (7) e (8) correspondem aos termos de correção de erros (EC). Os efeitos de longo prazo são calculados dividindo um coeficiente que, no EC, é associado a uma variável independente, com seu sinal invertido, pelo coeficiente que, no EC, é associado a um P/E. Esse cálculo é conhecido como transformação de Bårdsen (Bårdsen, 1989). A título de exemplo, o efeito de longo prazo do IMA-S é dado por $\frac{-\lambda_{2}}{\lambda_{1}}$.

A existência das relações em nível entre as variáveis, associada à existência de cointegração entre elas, pode ser avaliada conforme a abordagem do teste dos limites (bounds test), desenvolvida por Pesaran et al. (2001). Basicamente, testa-se a hipótese nula de os coeficientes $\lambda$ serem iguais a zero, o que equivale à ausência de cointegração, mediante valores de limites críticos providos para a comparação com uma estatística F. Pesaran et al. (2001) proveram os valores dos limites críticos assintóticos e Narayan (2005) proveu os valores desses limites para amostras finitas. O limite inferior assume que todas as variáveis são estacionárias, $I(0)$, e o limite superior assume que todas 
as variáveis são integradas em primeira ordem, $I(1)$. Se o valor da estatística F encontrada for inferior àquele do limite inferior, conclui-se que todas as variáveis são $I(0)$, portanto, não é possível haver cointegração. Por outro lado, se o valor da estatística $\mathrm{F}$ for superior àquele do limite superior, conclui-se que deve haver cointegração. Quando o valor da estatística $\mathrm{F}$ está entre o limite inferior e o limite superior, o teste é inconclusivo.

Cabe destacar que a abordagem ARDL de Pesaran e Shin (1998) e Pesaran et al. (2001) pode ser aplicada quando as variáveis são todas $I(1)$, todas $I(0)$, ou quando há uma mistura entre variáveis $I(1)$ e $I(0)$. Portanto, é facultativa a aplicação de testes de raiz unitária previamente à estimação desses modelos, em vista de eles não apresentarem o pressuposto de as variáveis terem que ser todas $I(1)$, como acontece na abordagem de análise de relações cointegradas de Johansen $(1988,1991)$. De qualquer modo, testes Dickey-Fuller Aumentado (ADF) e Kwiatkowski-Phillips-Schmidt-Shin (KPSS) aplicados sobre as variáveis deste estudo são apresentados no Apêndice. ${ }^{8}$

A abordagem ARDL de Pesaran et al. (2001) é recomendada quando as amostras de dados são pequenas, como é o caso da série temporal do P/E10, que apresenta 43 observações. Narayan (2005) proveu valores críticos do teste de cointegração de Pesaran et al. (2001) para amostras finitas de 30 a 80 observações. Adotando a abordagem em questão, o autor realizou análises empíricas da relação entre poupança e investimento com amostras de 43 e 47 observações. As séries do P/E1, dos retornos do IMA-S e aquelas de volatilidade nos mercados brasileiros de ações e de títulos, apresentam 163 observações. As estatísticas descritivas de todas as variáveis empregadas neste estudo são apresentadas no Apêndice.

\section{Resultados}

Inicialmente, é interessante observar o comportamento das séries temporais dos índices P/E1 e P/E10, assim como aquela dos retornos do IMA-S. Isso é ilustrado na Figura 2. É possível notar uma relação inversa entre o P/E1 e os retornos do IMA-S. Quando o P/E1 era baixo, os retornos do IMA$\mathrm{S}$, em termos de taxas de juros, eram altos; ou vice-versa. O coeficiente de correlação de Pearson entre essas variáveis é -0,49.

Essa relação parece ter se esvaído a partir do segundo trimestre de 2014. Em função disso, inicialmente, considerou-se uma dummy para captar o efeito da Crise Econômica Brasileira, ocorrida entre o segundo trimestre de 2014 e o último trimestre de 2016. Contudo, ao longo da modelagem econométrica,

${ }^{8}$ Para uma descrição desses testes de raiz unitária, veja Paterson (2011) e Kwiatkowski et al. (1992). 
Figura 2

Índice P/E1, índice P/E10 e retornos dos títulos públicos brasileiros

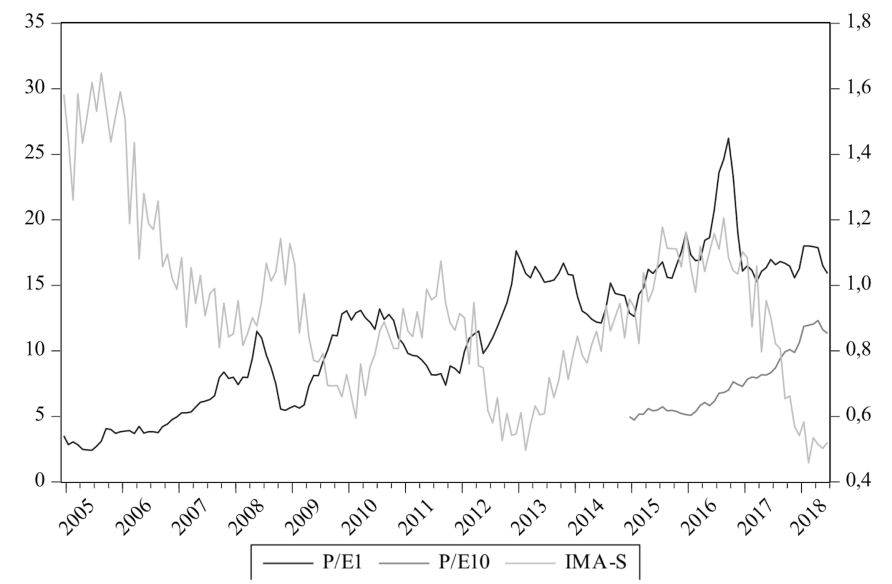

Nota: A haste da direita refere-se aos valores das taxas de juros — retornos dos títulos - e a haste da esquerda refere-se aos valores dos índices P/E.

notou-se que era mais adequado não inserir essa dummy, por ela apresentar um efeito conflitante com aquele da variável de volatilidade no mercado brasileiro de títulos públicos. O aumento dessa volatilidade, que, como evidenciado mais adiante, tem relação positiva com os P/Es, pode ter motivado o enfraquecimento da relação entre esses índices e o IMA-S durante o período da Crise Econômica Brasileira.

É mais difícil observar graficamente a relação entre o P/E10 e o IMA$\mathrm{S}$, devido ao curto período da série temporal do múltiplo. Mas, a partir de 2016, parece haver uma relação inversa entre essas variáveis. Enquanto o P/E10 aumentava, o IMA-S seguia uma trajetória de queda. O coeficiente de correlação de Pearson entre essas variáveis é $-0,86$.

Com essa impressão inicial a respeito das relações concernentes ao Modelo Fed no mercado financeiro brasileiro, prosseguiu-se, então, para a análise dos modelos ARDL/CECM.

\subsection{Modelo Fed Simples}

Primeiramente, foram estimados modelos ARDL correspondentes ao Modelo Fed Simples, conforme a especificação denotada pela Equação 5. No modelo que tem o P/E1 como variável dependente, foi definido um número máximo de 12 defasagens para cada variável, e a combinação ótima dessas defasagens foi selecionada automaticamente conforme o menor valor do Cri- 
Tabela 1

Estimações do modelo Fed Simples

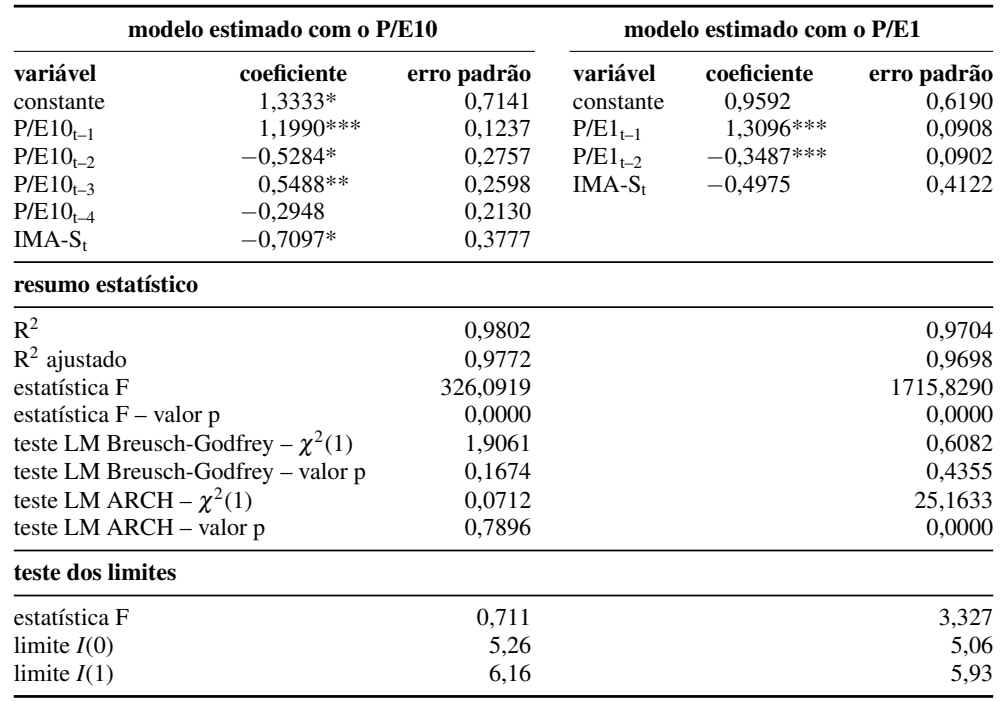

Nota: $* * * * * \mathrm{e}^{*}$ indicam coeficientes significativos aos níveis de $1 \%, 5 \%$ e $10 \%$ de significância, respectivamente. Em ambos os modelos foram adotados estimadores HAC. No modelo estimado com o P/E10, o número de observações ajustado é 39. No modelo estimado com o P/E1, o número de observações ajustado é 161 . No modelo estimado com o P/E10, foram adotados os valores críticos dos limites para amostras finitas de $n=40$, referentes ao nível de 5\% de significância, no teste de Pesaran et al. (2001). No modelo estimado com o P/E1, tais valores críticos são para $n=80$. Os testes sugerem ausência de cointegração. O período considerado no modelo estimado com o P/E10 foi abril de 2015 a junho de 2018, enquanto no modelo referente ao P/E1 foi fevereiro de 2005 a junho de 2018.

tério de Informação de Akaike (AIC). No modelo que tem o P/E10 como variável dependente, esse número máximo de defasagens teve que ser restrito a 5 , em virtude do número pequeno de observações na série temporal dessa variável. Em ambos os modelos, foram adotados estimadores consistentes com heterocedasticidade e autocorrelação (HAC) de Newey e West $(1987,1994)$. Em virtude de os testes dos limites sugerirem a não existência de cointegração entre as variáveis desses modelos, eles foram analisados na forma de ARDL. Os resultados das estimações do Modelo Fed Simples que relaciona o P/E1 e daquele que relaciona o P/E10 são apresentados na Tabela 1. Essa tabela também traz os testes dos limites de Pesaran et al. (2001).

Nos modelos ARDL exibidos na Tabela 1, observa-se que a variável IMAS não apresentou coeficientes significativos, considerando o nível de 5\% de significância. Portanto, pode-se constatar que o Modelo Fed em sua especificação mais simples não deve ser capaz de explicar o comportamento do P/E10 
e o comportamento do P/E1. Esse resultado corrobora Asness (2003), no que diz respeito à importância de considerar variáveis de volatilidade para que o Modelo Fed seja efetivo. Ele também está de acordo com Shiller (2015), que discorda da capacidade de as taxas de juros por si só preverem a trajetória de um P/E.

Os coeficientes de determinação $\left(\mathrm{R}^{2}\right)$ verificados nos dois modelos estimados são altos devido meramente aos valores defasados dos P/Es contribuírem muito para a explicação das trajetórias desses índices. Pode-se afirmar que há um considerável grau de persistência no comportamento deles.

Para ambos os modelos, os testes $\mathrm{F}$ convencionais rejeitam a hipótese nula de que as variáveis não contribuem para a explicação das variáveis dependentes; os testes de autocorrelação LM Breusch-Godfrey não rejeitam a hipótese nula de ausência de autocorrelação; e, como mencionado anteriormente, os testes dos limites de Pesaran et al. (2001) sugerem que não existem relações em nível - cointegração - entre as variáveis dos modelos, uma vez que as estatísticas F desses testes são inferiores aos limites críticos $I(0)$. No modelo estimado com o PE/10, o teste de heterocedasticidade LM ARCH não rejeitou a hipótese nula de ausência de heterocedasticidade condicional autorregressiva de primeira ordem. Por outro lado, no modelo estimado com o P/E1, esse teste rejeitou essa hipótese. Todavia, a adoção de estimadores HAC deve mitigar os efeitos da heterocedasticidade.

É importante destacar que os períodos dos modelos estimados neste estudo são distintos, uma vez que eles dependem do número de observações das séries históricas dos índices P/E1 e P/E10. Como mencionado, a série do P/E10 é menor porque esse índice apresenta uma média retroativa de 10 anos do indicador de lucros como divisor.

Como será observado mais adiante, ao incluir nos modelos as variáveis de volatilidade no mercado de títulos e no mercado de ações, evidencia-se a existência de cointegração, bem como o coeficiente da variável IMA-S passa a ser significativo. Essa mudança no resultado sinaliza que o Modelo Fed Simples sofre de viés de omissão. Isso explica os resultados de Estrada (2006), que mediante testes de cointegração, encontrou que a relação de equilíbrio de longo prazo correspondente ao Modelo Fed Simples existia somente em dois mercados financeiros entre aqueles de vinte países desenvolvidos. Os resultados desse autor devem ter sido influenciados pela não inclusão das variáveis de volatilidade.

\subsection{Modelo Fed Aumentado}

A partir da evidência de que as taxas de juros por si só não são capazes de explicar o comportamento dos índices $\mathrm{P} / \mathrm{E}$, avançou-se para a estimação 
dos modelos ARDL correspondentes ao Modelo Fed Aumentado. Seguindo Asness (2003), esses modelos incorporaram variáveis de volatilidade no mercado de ações e volatilidade no mercado de títulos, conforme a Equação 6. Porém, em virtude de os testes dos limites terem sugerido a existência de relações em nível - cointegração - entre as variáveis desses modelos, eles foram representados na forma de CECM, conforme a Equação 8. Os procedimentos de seleção de defasagens pelo AIC foram semelhantes aos dos modelos já apresentados. Além disso, também foram adotados estimadores HAC. Os resultados das estimações do Modelo Fed Aumentado que relaciona o P/E1 e daquele que relaciona o P/E10 são apresentados na Tabela 2.

Observa-se que, no longo prazo, tanto o IMA-S quanto o $\sigma_{\text {títulos }}$ apresentaram efeitos significativos sobre ambos os $\mathrm{P} /$ Es. A variável $\sigma_{\text {ações }}$ apresentou coeficiente apenas marginalmente significativo no modelo referente ao P/E1. Os sinais dessas variáveis estão de acordo com o que era esperado. Com embasamento em Asness (2003), esperava-se um sinal negativo para IMA-S, um sinal negativo para $\sigma_{\text {ações }}$ e um sinal positivo para $\sigma_{\text {títulos. }}$ O aumento dos retornos no mercado de títulos tende a fazer com que os investidores realoquem seus investimentos do mercado de ações para o mercado de títulos, de modo que, com a menor procura por ações, os preços caiam e, por conseguinte, os $\mathrm{P} / \mathrm{Es}$ também. O inverso pode ser dito sobre quando esses retornos diminuem. Por outro lado, um aumento da volatilidade no mercado de títulos tende a motivar os investidores a deslocarem seus investimentos do mercado de títulos para o mercado de ações, de modo a aumentar os P/Es. O inverso pode ser dito sobre quando essa volatilidade diminui. Cabe destacar que o forte aumento da volatilidade no mercado de títulos parece ter sido a explicação do rompimento da relação entre o P/E1 e os retornos dos títulos públicos durante a Crise Econômica Brasileira, ocorrida entre o segundo trimestre de 2014 e o último de 2016.

Em ambos os CECM referidos, houve variáveis especificadas automaticamente com defasagens ótimas iguais a zero. Quando isso acontece, elas não são incorporadas como defasagens em primeira diferença $(\Delta)$ no modelo, compondo somente o termo de correção de erros (EC). De acordo com a abordagem de Pesaran e Shin (1998) e Pesaran et al. (2001), essas variáveis devem ser interpretadas no contexto de uma decomposição $z_{t}=z_{t-1}+\Delta z_{t}$ de modo que possam ser incluídas no EC. Os mínimos quadrados estimados associados aos coeficientes dessas variáveis são estimativas simultâneas dos coeficientes de $z_{t-1}$ e $\Delta z_{t}$.

O R ${ }^{2}$ do CECM estimado com o P/E10 sugere que o Modelo Fed Aumentado apresenta um ajuste razoável. Por outro lado, com base no $\mathrm{R}^{2}$, pode-se

${ }^{9}$ Para mais, veja Pesaran e Shin (1998) e Pesaran et al. (2001). 
Tabela 2

Estimações do modelo Fed Aumentado

\begin{tabular}{|c|c|c|c|c|c|}
\hline \multicolumn{3}{|c|}{ modelo estimado com o P/E10 } & \multicolumn{3}{|c|}{ modelo estimado com o P/E1 } \\
\hline variável & coeficiente & erro padrão & variável & coeficiente & erro padrão \\
\hline constante & $6,2323 * * *$ & 1,9847 & constante & $3,0037 * * *$ & 0,5655 \\
\hline$\Delta \mathrm{P} / \mathrm{E} 10_{\mathrm{t}-1}$ & $0,5262 * * *$ & 0,1725 & $\Delta \mathrm{P} / \mathrm{E} 1_{\mathrm{t}-1}$ & $0,4205^{* * *}$ & 0,0703 \\
\hline$\Delta \mathrm{P} / \mathrm{E} 10_{\mathrm{t}-2}$ & $-0,1709$ & 0,2022 & $\Delta \sigma_{\text {títulos,t }}$ & $0,4867 * * *$ & 0,1401 \\
\hline$\Delta \mathrm{P} / \mathrm{E} 10_{\mathrm{t}-3}$ & $0,3932 * *$ & 0,1889 & $\Delta \sigma_{\text {títulos,t-1 }}$ & $0,3236 * * *$ & 0,1149 \\
\hline$\Delta$ IMA-S $_{\mathrm{t}}$ & $-4,2762$ & 6,6969 & & & \\
\hline$\Delta \mathrm{IMA}-\mathrm{S}_{\mathrm{t}-1}$ & 8,1612 & 6,6399 & & & \\
\hline$\Delta$ IMA-S $\mathrm{S}_{\mathrm{t}-2}$ & 4,0562 & 7,0202 & & & \\
\hline$\Delta \mathrm{IMA}-\mathrm{S}_{\mathrm{t}-3}$ & 9,4234 & 6,8488 & & & \\
\hline$\Delta \mathrm{IMA}-\mathrm{S}_{\mathrm{t}-4}$ & $13,2870 * *$ & 5,8627 & & & \\
\hline$\Delta \sigma_{\text {títulos,t }}$ & 0,3682 & 0,6391 & & & \\
\hline$\Delta \sigma_{\text {títulos,t-1 }}$ & $-0,5943$ & 0,6535 & & & \\
\hline$\Delta \sigma_{\text {títulos,t-2 }}$ & $-0,2396$ & 0,6911 & & & \\
\hline$\Delta \sigma_{\text {títulos,t-3 }}$ & $-0,7372$ & 0,6828 & & & \\
\hline$\Delta \sigma_{\text {títulos,t-4 }}$ & $-1,2256^{*}$ & 0,5975 & & & \\
\hline \multicolumn{3}{|c|}{ termo de correção de erros } & \multicolumn{3}{|c|}{ termo de correção de erros } \\
\hline $\mathrm{P} / \mathrm{E} 10_{\mathrm{t}-1}$ & $-0,4897$ *** & 0,1369 & $\mathrm{P} / \mathrm{E} 1_{\mathrm{t}-1}$ & $-0,1784 * * *$ & 0,0326 \\
\hline $\mathrm{IMA}_{\mathrm{A}-1}$ & $-9,8346^{* * * *}$ & 2,8755 & IMA-S $* * * *$ & $-2,5840 * * *$ & 0,5123 \\
\hline$\sigma_{\text {ações }} * * * *$ & 0,0000 & 0,0001 & $\sigma_{\text {ações, }} * * * *$ & $-0,0002 *$ & 0,0001 \\
\hline$\sigma_{\text {títulos, },-1}$ & $0,7046^{* * *}$ & 0,1920 & $\sigma_{\text {títulos,t-1 }}$ & $0,2867 * * *$ & 0,0614 \\
\hline \multicolumn{3}{|c|}{ relações de longo prazo } & \multicolumn{3}{|c|}{ relações de longo prazo } \\
\hline IMA-S & $-20,0814$ *** & 1,7178 & IMA-S & $-14,4818 * * *$ & 1,4527 \\
\hline$\sigma_{\text {ações }}$ & 0,0000 & 0,0003 & $\sigma_{\text {ações }}$ & $-0,0011 *$ & 0,0006 \\
\hline$\sigma_{\text {títulos }}$ & $1,4388 * * *$ & 0,1635 & $\sigma_{\text {títulos }}$ & $1,6070 * * *$ & 0,2141 \\
\hline \multicolumn{6}{|c|}{ resumo estatístico } \\
\hline $\mathrm{R}^{2}$ & & 0,6380 & & & 0,2886 \\
\hline estatística F & & 3,0210 & & & 15,8230 \\
\hline estatística F & & 0,0084 & & & 0,0000 \\
\hline teste $\mathrm{LM} \mathrm{Br}$ & dfrey $-\chi^{2}(1)$ & 0,0018 & & & 0,1477 \\
\hline teste $\mathrm{LM} \mathrm{Br}$ & dfrey - valor $\mathrm{p}$ & 0,9659 & & & 0,7008 \\
\hline teste LM Al & & 0,1374 & & & 9,3394 \\
\hline teste LM Al & or $\mathrm{p}$ & 0,7109 & & & 0,0022 \\
\hline \multicolumn{6}{|c|}{ teste dos limites } \\
\hline estatística F & & 4,358 & & & 8,2490 \\
\hline limite $I(0)$ & & 3,548 & & & 3,3630 \\
\hline limite $I(1)$ & & 4,803 & & & 4,5150 \\
\hline
\end{tabular}

Nota: $* * *, * * \mathrm{e}^{*}$ indicam coeficientes significativos aos níveis de $1 \%, 5 \%$ e $10 \%$ de significância, respectivamente. $* * * *$ denotam que foram selecionadas defasagens ótimas iguais a zero para as variáveis assinaladas, com base no AIC. Em ambos os modelos foram adotados estimadores HAC. No modelo estimado com o P/E10, o número de observações ajustado é 39. No modelo estimado com o P/E1, o número de observações ajustado é 161. No modelo estimado com o P/E10, foram adotados os valores críticos dos limites para amostras finitas de $n=40$, referentes ao nível de 5\% de significância, no teste de Pesaran et al. (2001). No modelo estimado com o P/E1, tais valores críticos são para $n=80$. Os testes sugerem existência de cointegração. O período considerado no modelo referente ao P/E10 foi abril de 2015 a junho de 2018, enquanto no modelo referente ao P/E1 foi fevereiro de 2005 a junho de 2018. 
afirmar que a capacidade de o referido modelo se ajustar ao P/E1 é baixa, ainda que, nele, as variáveis tenham apresentado coeficientes significativos. As estatísticas F convencionais dos modelos rejeitam a hipótese nula de que as variáveis não contribuem para a explicação das variáveis dependentes; os testes de autocorrelação LM Breusch-Godfrey não rejeitam a hipótese nula de ausência de autocorrelação. Quanto aos testes de heterocedasticidade LM $\mathrm{ARCH}$, aquele referente ao modelo estimado com o P/E10 não rejeita a hipótese nula de ausência de heterocedasticidade, enquanto aquele referente ao modelo estimado com o P/E1 rejeitou essa hipótese. A adoção de estimadores HAC deve mitigar os efeitos desse problema de heterocedasticidade.

Quanto aos testes dos limites de Pesaran et al. (2001), no caso do CECM que relaciona o índice $\mathrm{P} / \mathrm{E} 1$, a estatística $\mathrm{F}$ encontrada foi superior ao limite $I(1)$, sugerindo a existência de relações em nível - cointegração - entre as variáveis. Contudo, no caso do CECM que relaciona o P/E10, considerando os valores críticos para os limites $I(0)$ e $I(1)$ ao nível de $5 \%$ de significância, o valor da estatística $\mathrm{F}$ se encontrou entre aqueles de tais limites, caso em que o resultado do teste é inconclusivo. Uma alternativa a esse resultado inconclusivo foi comparar a estatística $\mathrm{F}$ com os valores críticos referentes ao nível de $10 \%$ de significância, a saber: limite $I(0)$ igual a 2,72 e limite I(1) igual a 3,77. Nesse caso, a estatística F encontrada, que é igual a 4,358, foi superior ao limite $I(1)$, sugerindo a existência de relações em nível cointegração - no modelo que relaciona o índice P/E10. Além disso, se considerados os limites críticos assintóticos $-\mathrm{n}=1.000-$, a estatística $\mathrm{F}$ ficaria um pouco acima do limite crítico $I(1)$ cujo valor é 4,350 ao nível de 5\% de significância, também sugerindo a existência de relações em nível. Cabe destacar que as relações entre as variáveis podem se tornar mais evidentes na medida em que a série do P/E10, que apresenta poucas observações, for expandida com dados mais recentes.

Uma terceira evidência que corrobora a existência de cointegração entre as variáveis do CECM com o $\mathrm{P} / \mathrm{E} 10$ provém do $t$-bounds test, que compara a estatística $t$ de um modelo de correção de erros (ECM) convencional que difere do modelo de correção de erros condicional (CECM) - com os valores críticos de Pesaran et al. (2001). Nesse teste a estatística $t$ de valor 4,46 foi superior ao limite $I(1)$ para o nível de 5\% significância, de valor 3,78, sugerindo a existência de relações em nível — cointegração. ${ }^{10}$

Com base nos resultados encontrados mediante a estimação de modelos conforme as especificações do Modelo Fed Simples e do Modelo Fed Aumentado, observou-se que a consideração de variáveis de volatilidade nos mercados de títulos é fundamental para que o Modelo Fed seja efetivo no

${ }^{10}$ Para mais sobre o t-bounds test, veja Pesaran et al. (2001). 
contexto do mercado financeiro brasileiro. Isso corrobora a ideia de Asness (2003) de que o risco deve influenciar as decisões dos investidores a respeito da alocação do capital no mercado de títulos ou no mercado de ações. Todavia, não se observou um efeito significativo, considerando o nível de 5\% de significância, para a volatilidade no mercado de ações, somente para a volatilidade no mercado de títulos. A ideia de Shiller (2015) sobre o Modelo Fed não ser capaz de explicar a trajetória dos P/Es é coerente com a evidência empírica somente quando a especificação desse modelo considera apenas os retornos no mercado de títulos públicos, em termos de taxas de juros. No que diz respeito aos efeitos de curto prazo, parece não haver resultados claros, tendo em vista que esses efeitos variaram consideravelmente entre os modelos com distintos índices P/E. Por outro lado, evidenciaram-se relações de longo prazo entre os retornos e a volatilidade no mercado brasileiro de títulos e os índices P/E. A relação entre os retornos do mercado brasileiro de títulos e os P/Es é negativa, e a relação entre a volatilidade no mercado brasileiro de títulos e os P/Es é positiva. Esses resultados estão de acordo com a teoria a respeito do Modelo Fed.

\section{Considerações finais}

A análise empírica sugeriu que o Modelo Fed Aumentado, de fato, pode contribuir para a explicação dos índices $\mathrm{P} / \mathrm{E}$. Os investidores parecem considerar o investimento no mercado de títulos como uma alternativa ao investimento no mercado de ações. Eles também devem considerar o risco nesses distintos mercados na tomada de decisão sobre em qual deles investir seu capital. Isso faz sentido, especialmente, no Brasil, que historicamente tem apresentado taxas de juros muito mais altas do que aquelas praticadas em países como os Estados Unidos.

Uma contribuição prática deste estudo decorre da evidência de que uma queda das taxas de juros tende a conduzir a uma valorização do mercado de ações. Se esse efeito, de fato, é motivado por um fluxo de investimentos que parte do mercado de títulos para o mercado de ações, o que tende a conduzir ao aumento dos preços das ações, investidores podem tirar proveito desses momentos no estabelecimento de estratégias de investimento. Por outro lado, em momentos nos quais uma possível sobrevalorização do mercado de ações tende a ser corrigida mediante quedas bruscas nos preços, deve ser oportuno deslocar os investimentos do mercado de ações para o mercado de títulos, no intuito de resguardar temporariamente o capital. Os índices P/E demasiadamente altos podem ser considerados indícios de que tais eventos estão próximos de ocorrer, tendo em vista que os preços das ações não devem romper continuamente a relação com os lucros das empresas (Campbell e 
Shiller, 1998). As transações devem ser estabelecidas em termos de uma carteira ampla de ações ou cotas de um exchange traded fund (ETF), de modo a acompanhar a composição do Ibovespa - a mesma dos índices P/E.

Por fim, vários estudos futuros que se utilizem dos índices $\mathrm{P} / \mathrm{E}$ podem ser sugeridos. São incentivados aqueles que analisem a relação entre tais $\mathrm{P} /$ Es e o volume transacionado em ações na bolsa de valores. Uma hipótese interessante é de que os índices $\mathrm{P} / \mathrm{E}$ tenham apresentado uma tendência positiva em decorrência do aumento do volume de investimentos na bolsa brasileira, tendo em vista uma relação positiva entre preços de ações e volume de negociações já evidenciada na literatura (Saatcioglu e Starks, 1998; Gündüz e Hatemi-J, 2005). Sugerem-se, ainda, estudos que relacionem os índices P/E com indicadores de incerteza da economia. Nesse caso, uma hipótese que pode ser testada consiste em uma relação negativa entre os P/Es e algum indicador de incerteza, tendo embasamento na ideia de que os investidores optariam por não manter seus investimentos em ações em momentos de alta incerteza econômica, impactando os P/Es. Uma última sugestão para estudos futuros é assumir os P/Es como indicadores do nível de otimismo dos investidores do mercado de ações e investigar assimetria de informações com base em algum indicador de confiança dos empresários.

\section{Agradecimentos}

Os autores agradecem a Aureliano Angel Bressan, Márcio Antônio Salvato e Robert Aldo Iquiapaza Coaguila, além de ao editor associado Marco Túlio Lyrio, pelos comentários que contribuíram para o aperfeiçoamento do artigo.

\section{Referências}

Almon, S. (1965). The distributed lag between capital appropriations and expenditures, Econometrica 33(1): 178-196.

Asness, C. S. (2000). Stocks versus bonds: Explaining the equity risk premium, Financial Analysts Journal 56(2): 96-113.

Asness, C. S. (2003). Fight the Fed Model, Journal of Portfolio Management 30(1): 11-24.

Bårdsen, G. (1989). Estimation of long run coefficients in error correction models, Oxford Bulletin of Economics and Statistics 51(3): 345-350.

BM\&FBovespa (2014). Manual de definições e procedimentos dos índices da BM\&FBovespa. Recuperado em 08 fevereiro 2019. 
URL: http: / / bvmf . bmf bovespa.com.br/indices/downloa d/Manual-de-procedimentos-pt-br.pdf

Campbell, J. Y. e Shiller, R. J. (1998). Valuation ratios and the long-run stock market outlook, Journal of Portfolio Management 24(2): 11-26.

Castro, F. H., Eid Junior, W., Santana, V. F. e Yoshinaga, C. E. (2019). Fiftyyear history of the Ibovespa, Revista Brasileira de Finanças 17(3): 47-65.

Engle, R. F. e Granger, C. W. J. (1987). Co-integration and error correction: Representation, estimation, and testing, Econometrica 55(2): 251-276.

Estrada, J. (2006). The Fed model: A note, Finance Research Letters 3(1): 14-22.

Graham, B. e Dodd, D. L. (1934). Security Analysis, 1. edn, McGraw-Hill, New York.

Granger, C. W. J. e Newbold, P. (1974). Spurious regressions in econometrics, Journal of Econometrics 2(2): 111-120.

Gündüz, L. e Hatemi-J, A. (2005). Stock price and volume relation in emerging markets, Emerging Markets Finance and Trade 41(1): 29-44.

Johansen, S. (1988). Statistical analysis of cointegration vectors, Journal of Economic Dynamics \& Control 12(2): 231-254.

Johansen, S. (1991). Estimation and hypothesis testing of cointegration vectors in Gaussian vector autoregressive models, Econometrica 59(6): 15511580 .

Kwiatkowski, D., Phillips, P. C. B., Schmidt, P. e Shin, Y. (1992). Testing the null hypothesis of stationarity against the alternative of a unit root, Journal of Econometrics 54(1-3): 159-178.

Narayan, P. K. (2005). The saving and investment nexus for china: Evidence from cointegration tests, Applied Economics 37(17): 1979-1990.

Newey, W. K. e West, K. D. (1987). A simple, positive semi-definite, heteroskedasticity and autocorrelation consistent covariance matrix, Econometrica 55(3): 703-708.

Newey, W. K. e West, K. D. (1994). Automatic lag selection in covariance matrix estimation, The Review of Economic Studies 61(4): 631-653. 
Paterson, K. (2011). Unit Roots Tests in Time Series, Palgrave Macmillan, Basingstoke.

Pesaran, M. H. e Shin, Y. (1998). An autoregressive distributed-lag modelling approach to cointegration analysis, Econometric Society Monographs 31: 371-413.

Pesaran, M. H., Shin, Y. e Smith, R. J. (2001). Bounds testing approaches to the analysis of level relationships, Journal of Applied Econometrics 16(3): 289-326.

Saatcioglu, K. e Starks, L. T. (1998). The stock price-volume relationship in emerging stock markets: The case of Latin America, International Journal of Forecasting 14(2): 215-225.

Schmidt, P. (1974). A Modification of the Almon distributed lag, Journal of the American Statistical Association 69(347): 679-681.

Shiller, R. J. (1989). Market Volatility, MIT Press, London / New York.

Shiller, R. J. (2005). Irrational Exuberance, 2. edn, Princeton University Press, Princeton.

Shiller, R. J. (2015). Irrational Exuberance (Capítulo 2), 3. edn, Princeton University Press, Princeton.

S\&P Dow Jones Indices (2018). Index mathematics methodology. Recuperado em 08 de fevereiro de 2019.

URL: https: //us.spindices.com/documents/methodolo gies/methodology-index-math.pdf

Weigand, R. A. e Irons, R. (2008). Compression and expansion of the market P/E ratio: The Fed Model explained, Journal of Investing 17(1): 55-64. 


\section{A. Apêndice}

As tabelas A1 e A2 apresentam as estatísticas descritivas das variáveis utilizadas neste artigo. As estatísticas descritivas de amostras comuns permitem comparar o valor de algumas variáveis em virtude de serem referentes a um período padronizado, enquanto as estatísticas descritivas de amostras individuas são referentes às séries temporais completas em períodos distintos.

\section{Tabela A1}

Estatísticas descritivas de amostras comuns

\begin{tabular}{lccccc}
\hline estatísticas & P/E10 & P/E1 & IMA-S & $\sigma_{\text {títulos }}$ & $\sigma_{\text {ações }}$ \\
\hline média & 7,538717 & 17,34099 & 0,915185 & 9,514026 & 1398,392 \\
máximo & 12,30714 & 26,20351 & 1,205469 & 12,6727 & 3208,39 \\
mínimo & 4,70978 & 12,62215 & 0,458122 & 5,643138 & 454,4341 \\
desvio padrão & 2,363537 & 2,748808 & 0,225766 & 1,933637 & 567,0174 \\
observações & 43 & 43 & 43 & 43 & 43 \\
\hline
\end{tabular}

Nota: O período comum às amostras considerado é de dezembro de 2014 a junho de 2018.

Tabela A2

Estatísticas descritivas de amostras individuais

\begin{tabular}{lccccc}
\hline estatísticas & P/E10 & P/E1 & IMA-S & $\sigma_{\text {títulos }}$ & $\sigma_{\text {ações }}$ \\
\hline média & 7,538717 & 11,39175 & 0,931149 & 6,026142 & 1354,003 \\
máximo & 12,30714 & 26,20351 & 1,648062 & 12,6727 & 4671,665 \\
mínimo & 4,70978 & 2,413549 & 0,458122 & 3,242793 & 322,9336 \\
desvio padrão & 2,363537 & 5,24229 & 0,259595 & 2,470685 & 630,3999 \\
observações & 43 & 163 & 163 & 163 & 163 \\
período & $12 / 2014 \mathrm{a}$ & $12 / 2004 \mathrm{a}$ & $12 / 2004 \mathrm{a}$ & $12 / 2004 \mathrm{a}$ & $12 / 2004 \mathrm{a}$ \\
& $06 / 2018$ & $06 / 2018$ & $06 / 2018$ & $06 / 2018$ & $06 / 2018$ \\
\hline
\end{tabular}

Os resultados dos testes de raiz unitária ADF e KPSS aplicados sobre as variáveis usadas neste artigo são mostrados nas tabelas A3 e A4, a seguir. A utilização da abordagem ARDL/CECM de Pesaran et al. (2001) se deu em virtude de o conjunto de séries temporais utilizado apresentar tanto séries $I(0)$ quanto séries $I(1)$.

As tabelas A5 e A6, a seguir, apresentam as regressões lineares estáticas, estimadas por Mínimos Quadrados Ordinários (MQO), referentes às duas especificações do Modelo Fed adotadas neste artigo. Nesses modelos, observaram-se estatísticas Durbin-Watson muito baixas, e os testes de autocorrelação LM Breusch-Godfrey rejeitaram a hipótese nula de ausência de autocorrelação. Portanto, há um grande risco de tratar-se de regressões espúrias. 
Tabela A3

Testes de raiz unitária Dickey-Fuller aumentado

\begin{tabular}{llcrrr}
\hline variável & especificação & defasagens & estat. do teste & valor crítico & resultado \\
\hline P/E10 & constante & 4 & 0,6928 & $-2,9411$ & $I(1)$ \\
P/E10 & constante e tendência & 4 & $-2,3116$ & $-3,5331$ & $I(1)$ \\
P/E1 & constante & 1 & $-2,0416$ & $-2,8794$ & $I(1)$ \\
P/E1 & constante e tendência & 1 & $-4,0410$ & $-3,4380$ & $I(0)$ \\
IMA-S & constante & 1 & $-1,7660$ & $-2,8794$ & $I(1)$ \\
IMA-S & constante e tendência & 12 & $-3,2202$ & $-3,4401$ & $I(1)$ \\
$\sigma_{\text {títulos }}$ & constante & 12 & $-1,7290$ & $-2,8807$ & $I(1)$ \\
$\sigma_{\text {títulos }}$ & constante e tendência & 13 & $-3,2655$ & $-3,4403$ & $I(1)$ \\
$\sigma_{\text {ações }}$ & constante & 8 & $-2,8275$ & $-2,8802$ & $I(1)$ \\
$\sigma_{\text {ações }}$ & constante e tendência & 8 & $-2,7992$ & $-3,4393$ & $I(1)$ \\
\hline
\end{tabular}

Nota: Os valores críticos são referentes ao nível de 5\% de significância.

\section{Tabela A4}

Testes de raiz unitária Kwiatkowski-Phillips-Schmidt-Shin

\begin{tabular}{llcrrr}
\hline variável & especificação & Bandwidth & estat. do teste & valor crítico & resultado \\
\hline P/E10 & constante & 5 & 0,7635 & 0,4630 & $I(1)$ \\
P/E10 & constante e tendência & 5 & 0,1856 & 0,1460 & $I(1)$ \\
P/E1 & constante & 10 & 1,4118 & 0,4630 & $I(1)$ \\
P/E1 & constante e tendência & 9 & 0,0517 & 0,1460 & $I(0)$ \\
IMA-S & constante & 10 & 0,5392 & 0,4630 & $I(1)$ \\
IMA-S & constante e tendência & 10 & 0,2141 & 0,1460 & $I(1)$ \\
$\sigma_{\text {títulos }}$ & constante & 10 & 1,0264 & 0,4630 & $I(1)$ \\
$\sigma_{\text {títulos }}$ & constante e tendência & 10 & 0,1713 & 0,1460 & $I(1)$ \\
$\sigma_{\text {ações }}$ & constante & 8 & 0,2319 & 0,4630 & $\mathrm{I}(0)$ \\
$\sigma_{\text {ações }}$ & constante e tendência & 8 & 0,1719 & 0,1460 & $I(1)$ \\
\hline
\end{tabular}

Nota: Os valores críticos são referentes ao nível de 5\% de significância. 


\section{Tabela A5}

Estimações do modelo Fed Simples por MQO

\begin{tabular}{lcrlrr}
\hline & \multicolumn{2}{c}{ modelo estimado com o P/E10 } & & modelo estimado com o P/E1 \\
\cline { 2 - 3 } variável & coeficiente & erro padrão & & coeficiente & erro padrão \\
\hline constante & $15,8116^{* * * *}$ & 0,7931 & & $20,6943^{* * *}$ & 1,5982 \\
IMA-S & $-9,0395^{* * * *}$ & 0,9102 & & $-9,9904^{* * *}$ & 2,0967 \\
\hline resumo estatístico & & & & 0,2447 \\
\hline $\mathrm{R}^{2}$ & 0,7456 & & 52,1727 \\
estatística F & 120,1392 & & 0,0000 \\
estatística F - valor $\mathrm{p}$ & 0,0000 & & 0,0924 \\
estatística Durbin-Watson & 0,5991 & & 148,2993 \\
teste LM Breusch-Godfrey $-\chi^{2}(1)$ & 19,4683 & & 0,0000 \\
teste LM Breusch-Godfrey - valor $\mathrm{p}$ & 0,0000 & &
\end{tabular}

Nota: $* * *, * * \mathrm{e} *$ indicam coeficientes significativos aos níveis de $1 \%, 5 \%$ e $10 \%$ de significância, respectivamente. Em ambos os modelos foram adotados estimadores HAC. No modelo estimado com o P/E10, o número de observações é 43, enquanto, no modelo estimado com o P/E1, o número de observações é 163 . O período considerado no modelo referente ao P/E10 foi dezembro de 2014 a junho de 2018, enquanto aquele considerado no modelo referente ao P/E1 foi dezembro de 2004 a junho de 2018.

\section{Tabela A6}

\section{Estimações do modelo Fed Aumentado por MQO}

\begin{tabular}{|c|c|c|c|c|}
\hline \multirow[b]{2}{*}{ variável } & \multicolumn{2}{|c|}{ modelo estimado com o P/E10 } & \multicolumn{2}{|c|}{ modelo estimado com o P/E1 } \\
\hline & coeficiente & erro padrão & coeficiente & erro padrão \\
\hline constante & $12,5315^{* * * *}$ & 0,9534 & $14,0211 * * *$ & 1,5957 \\
\hline IMA-S & $-18,1234 * * *$ & 1,5876 & $-12,8334 * * *$ & 1,0477 \\
\hline$\sigma_{\text {ações }}$ & 0,0002 & 0,0002 & $-0,0005^{* *}$ & 0,0003 \\
\hline$\sigma_{\text {títulos }}$ & $1,1914 * * *$ & 0,1568 & $1,6650^{* * * *}$ & 0,1615 \\
\hline \multicolumn{5}{|l|}{ resumo estatístico } \\
\hline \multicolumn{2}{|l|}{$\mathrm{R}^{2}$} & 0,9286 & & 0,8427 \\
\hline \multicolumn{2}{|l|}{ estatística F } & 169,1897 & & 283,9706 \\
\hline \multicolumn{2}{|l|}{ estatística F - valor p } & 0,0000 & & 0,0000 \\
\hline \multicolumn{2}{|c|}{ estatística Durbin-Watson } & 1,0681 & & 0,3098 \\
\hline \multicolumn{2}{|c|}{ teste LM Breusch-Godfrey $-\chi^{2}(1)$} & 9,7343 & & 115,5739 \\
\hline \multicolumn{2}{|c|}{ teste LM Breusch-Godfrey - valor p } & 0,0018 & & 0,0000 \\
\hline
\end{tabular}

***, ** e * indicam coeficientes significativos aos níveis de $1 \%, 5 \%$ e $10 \%$ de significância, respectivamente. Em ambos os modelos foram adotados estimadores HAC. No modelo estimado com o P/E10, o número de observações é 43, enquanto, no modelo estimado com o P/E1, o número de observações é 163 . O período considerado no modelo referente ao P/E10 foi dezembro de 2014 a junho de 2018, enquanto aquele considerado no modelo referente ao P/E1 foi dezembro de 2004 a junho de 2018. 